\title{
Theoretical study of hydrogen adsorption on graphene nanostructures functionalized with nickel for solid state hydrogen storage
}

\author{
Issa Zainalabdeen Hassan ${ }^{1 *}$, Ameer Hawas Khorsheed ${ }^{2}$ \\ ${ }^{1 *, 2}$ Department of physics, college of education for pure sciences, Kirkuk University, Kirkuk, Iraq \\ E-mail: ${ }^{1 *}$ Issa.assaflly@gmail.com, ${ }^{2}$ Frzn2013@yahoo.com
}

(Received September 16, 2020; Accepted November 14, 2020; Available online June 01, 2021)

DOI: 10.33899/edusj.2020.128376.1112, (c) 2021, College of Education for Pure Science, University of Mosul.

This is an open access article under the CC BY 4.0 license (http://creativecommons.org/licenses/by/4.0/).

\begin{abstract}
Hydrogen adsorption and storage on nickel- activated, pure graphene and boron-doped graphene was study using density functional theory simulations based on generalized gradient approximation methods (DFT-GGA). It was found that the nickel atoms tend to clustering on the surface of pure graphene due to the high cohesive energy of nickel compared to the energy of nickel binding to the surface of pure graphene, which decrease the storage capacity of hydrogen. It was also found that the storage capacity of seven hydrogen molecules on pure and activated graphene with a nickel atom is equal to $(10.2 \mathrm{wt} . \%)$ With an average binding energy $(0.27 \mathrm{eV})$, and the storage capacity for the same number of hydrogen molecules is (11.3 wt.\%) With an average binding energy $(0.22 \mathrm{eV})$, This indicates that the adsorption process will take place at ambient conditions. The process of inoculating graphene-doped boron and nickel activating is an effective strategy for improving the average binding energies and the storage capacity of hydrogen molecules in the graphene nanostructures.
\end{abstract}

Keywords: Hydrogen storage, adsorption, Graphene, nickel and binding energy.

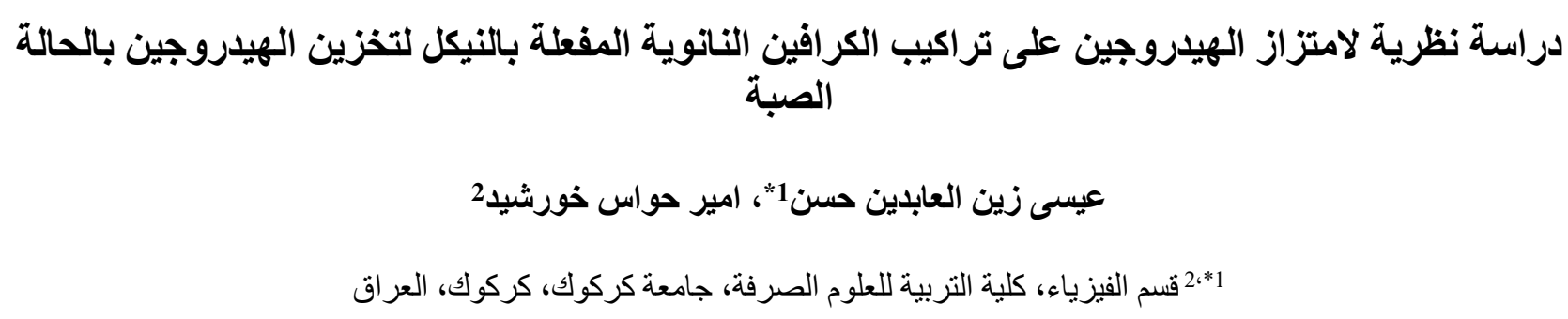

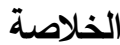

تمت دراسة امتزاز الهيدروجين وتخزينه على الكرافين النقي والمفعل بالنيكل مرة والكرافين المطعم بالبورون والهفعل بالنيكل مرة

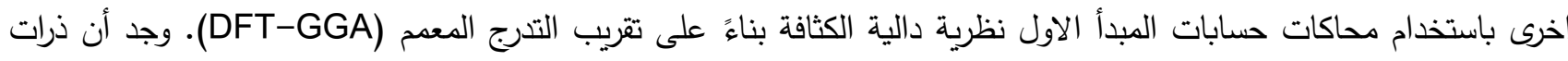
النيكل تميل الى التكتل على سطح الكرافين النقي بسبب طاقة التلاصق العالية للنيكل بالمقارنة مع طاقة ارتباط النيكل مع سطح

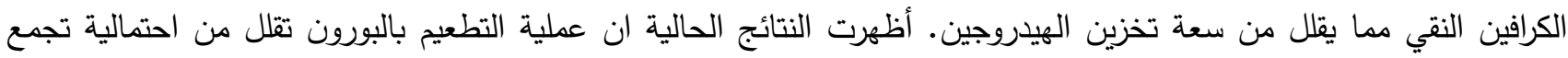
ذرات النيكل من خلال زيادة طاقة ارتباط ذرات النيكل مع سطح الكرافين. كما وجد ان سعة التخزين لسبع جزيئات هيدروجين فوق

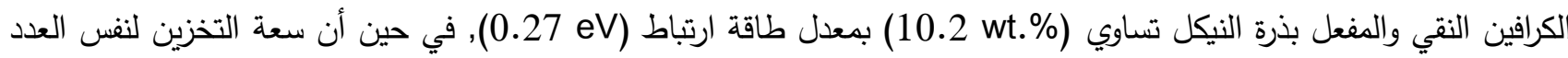
من جزيئات الهيدروجين هو (11.3 wt.\%) بمعدل طاقة ربط (0.22 eV), مما يدل على ان عملية الامتزاز سوف تحدث ضمن 
الظروف المحيطة. وان عملية تطعيم الكرافين بالبورون وتفعيل سطحه بالنيكل تعتبر استراتيجية فعالة لتحسين معدل طاقات الارتباط وسعة تخزين جزيئات الهيدروجين في تراكيب الكرافين النانوية.

الكلمات المفتاحية: خزن الهيدروجين, الامتزاز , كرافين, نيكل وطاقة الارتباط.

Introduction المقدمة

يعتبر العيدروجين وقودا واعدا وصديقا للبيئة لأنه يمتلك طاقة عالية نسبة الى الكتلة ويمكنه انتاج طاقة نظيفة خالية من المخلفات الكربونية الضارة, إذ ان كمية الطاقة المتحررة للكيلوغرام الواحد من الهيدروجين تعادل

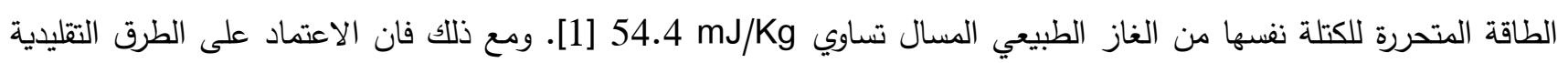

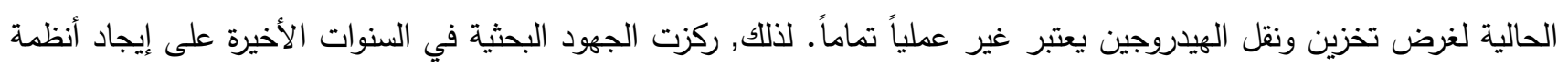

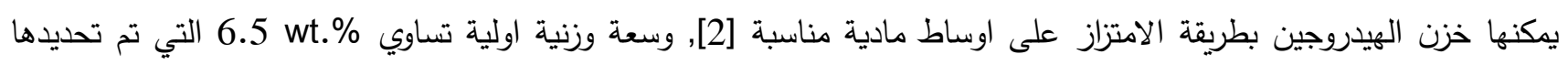

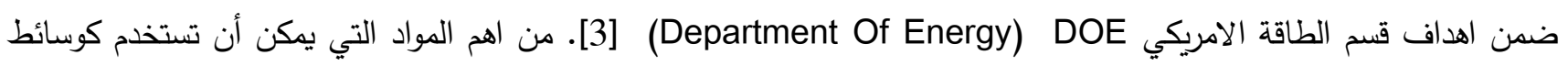
امتزاز فعال لجزيئات الهيدروجين هي الهياكل الكربونية النانوية ، نظرًا لوزنها المنخفض نسبيًا ومساحاتها السطحية العالية [4]. يعتبر الكرافين من افضل صور الكربون المعروفة بسبب امتلاكه طبقة ذرية أحادية بسمك ذرة كربون واحدة ذات مساحة سطحية

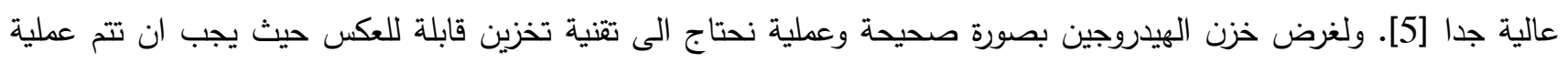

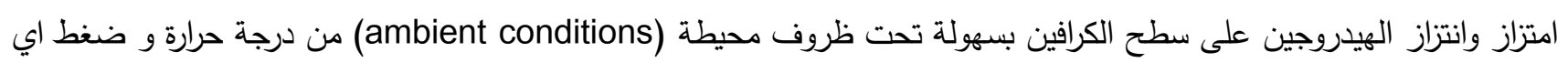
ان لا يكون ارتباط الهيدروجين قوي جدا ولا ضعيف جدا. بناءً على هذه المتطلبات العملية، وجد ان نطاق طاقة الربط المثالية

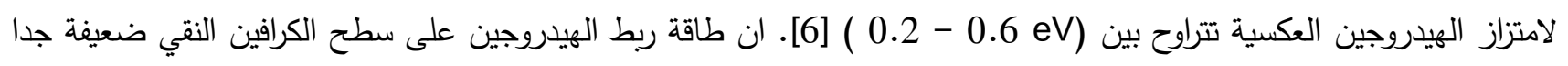

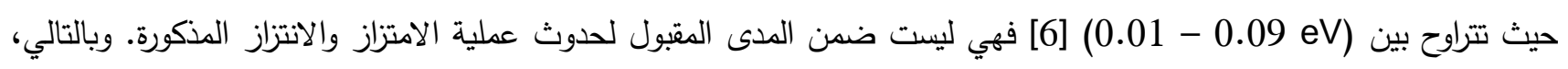
فإن العديد من الدراسات النظرية اقترحت تعديلات عدة على الكرافين لتحسين قدرته التخزينية. ومن هذه التعديلات تفعيل سطح الكرافين بذرات المعادن ودراسة طاقة ارتباطها وموقعها المفضل على سطح الكرافين بالإضافة الى عملية التطعيم من خلال استبدال

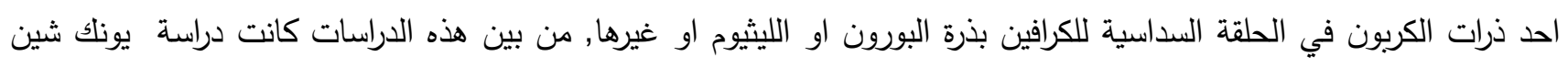

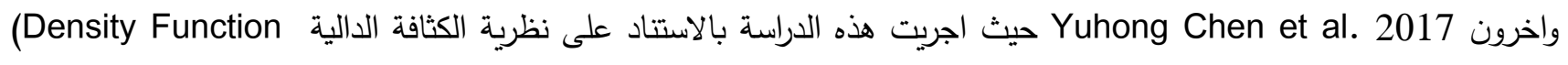
ونتائج الدراسة بينت ان الموقع المفضل والاكثر استقرارا لذرة السكانديوم (Sc) هو فوق مركز حلقة الكربون على (DFT)( DFT) سطح الكرافين, كما بينت هذه الدراسة ان اقصى عدد من جزيئات الهيدروجين (H2) التي يمكن امتزازها هي أربع جزيئات بمعدل طاقة امتزاز (0.429 eV). وامكانية تحسين سعة تخزين الهيدروجين للنظام على نحو فعال بإضافة ذرة Sc على كلى الجانب الاخر

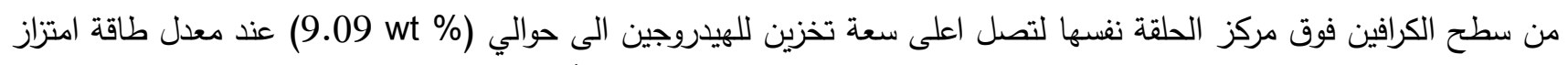

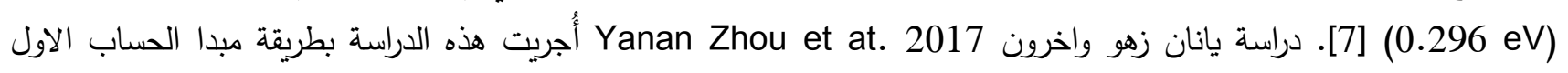
باستخدام برنامج (CASTEP) على اساس نظرية الكثافة الدالية (DFT) حيث تم دراسة خصائص امتزاز (Frist Principle) الهيدروجين على سطح خلية الكرافين التي تحتوي على عيوب احادية وثنائية وثلاثية ورباعية ومطعمة بذرة الليثيوم (Li) و مدتزة

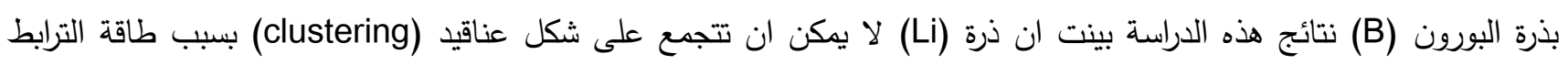

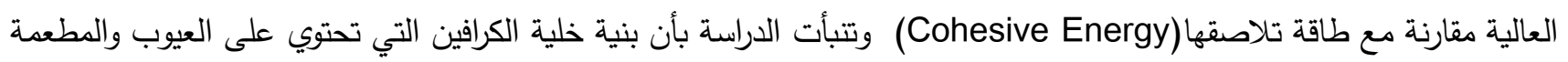

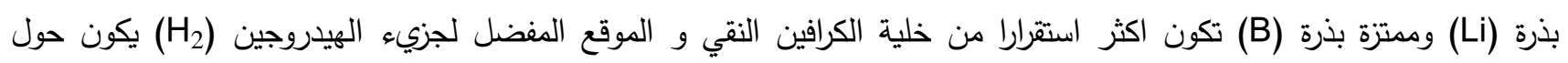

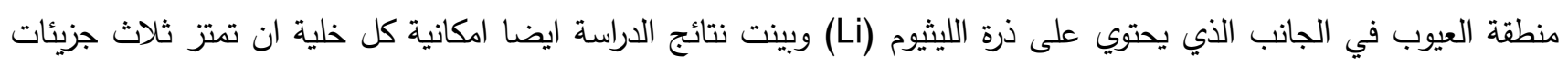

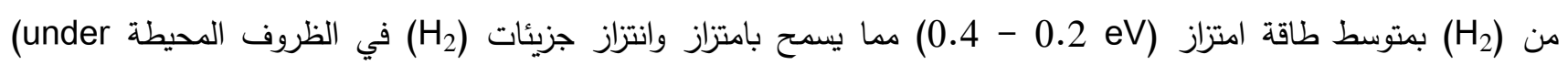


كما توقعت هذه الدراسة بان يكون الكرافين المطعم بمادة الليثيوم (Li) وممتز بذرات (B) وسطا واعدا لتخزين الهيدروجين [8]. دراسة يونلي وانج واخرون Yunlei Wang et al. 2020 استخدمت في هذه الدراسة مبدأ الحسابات

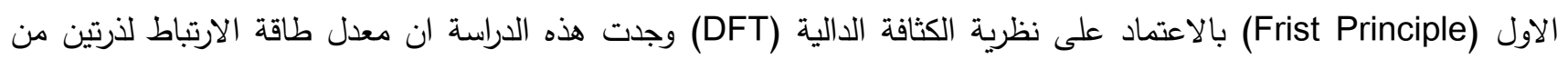

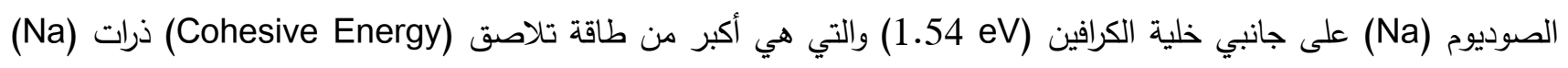
التي تساوي (eV)

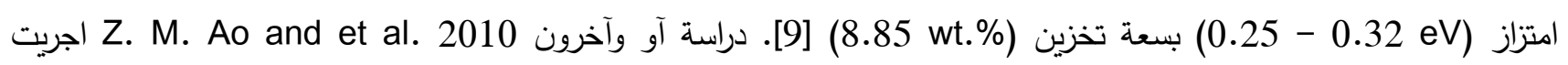

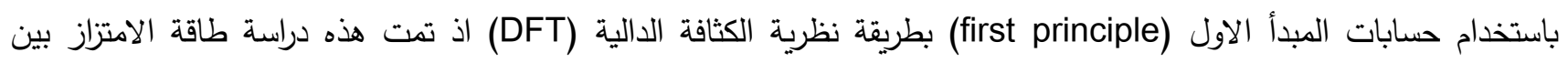
جزيء الهيدروجين (H2) وسطح الكرافين النقي و الممتز بذرة النيتروجين (N) حيث بينت هذه الدراسة الموقع المفضل لامتزاز ذرة

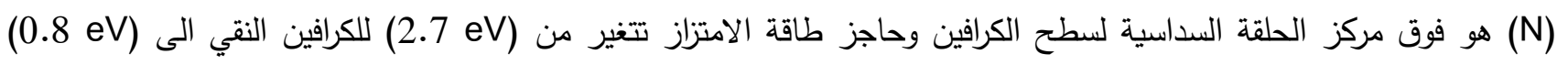

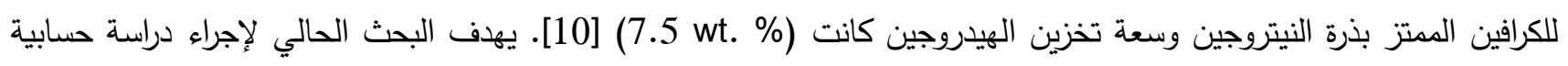
لكفاءة امتزاز جزيئات الهيدروجين على سطح الغرافين النقي والمطعم بالبورون (B) والمفعل بذرة النيكل (Ni) وحساب معدل طاقة لئل

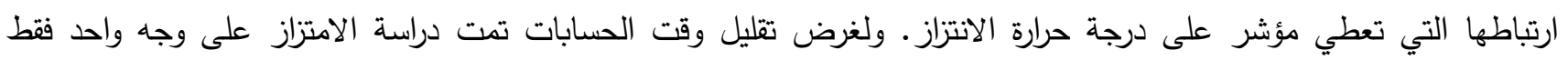
لغرض المقارنة مع الدراسات المشابهة.

\section{Computational Method طريقة الحساب}

هناك طريقتان أساسيتان لدراسة تركيب الجزيئات وتفاعلاتها، متمثلة بطريقة الميكانيك الجزيئية (Molecular Mechanics) ونظرية التركيب الاكتروني (Electron Structure (Methods) تعتمد طريقة الميكانيك الجزيئية في حساباتها على قوانين الفيزياء الكلاسيكية وعادة يتم استخدامها لحساب تركيب وخصائص الجزيئات [11]. أما طريقة نظرية التركيب الآكتروني فقد تم

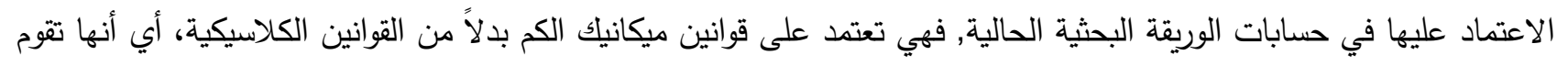

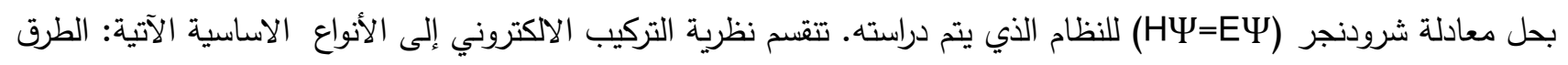

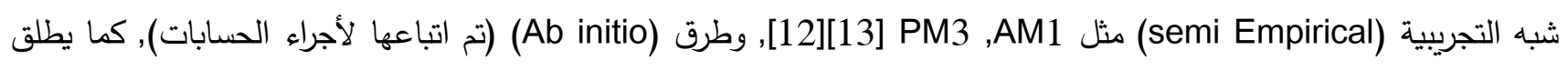

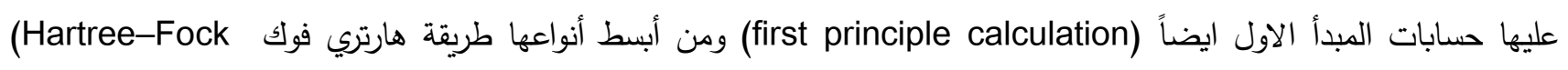

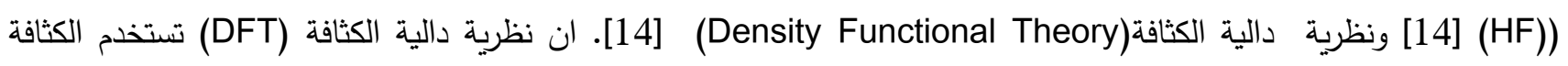
الإكترونية لغرض الحصول على حلول عددية لمعادلة شرودنجر للنظام عن طريق هو إعادة صياغة المسالة الكمومية وتحويلها من مسالة نظام متعدد الجسيمات إلى مسالة أحادية الجسيمة عن طريق التخلي عن التعامل مع دالة الموجة والاستعانة بدالية الكثافة

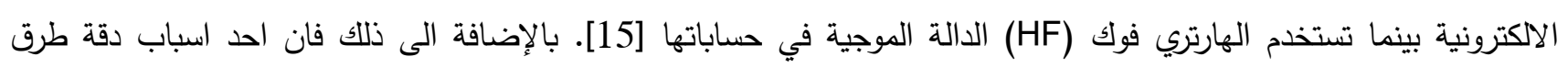

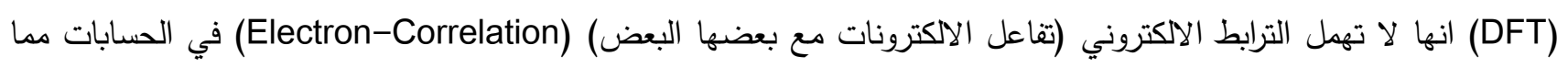
يجعلها اكثر تكلفة حسابيا من طرق هارتري فوك (HF) والطرق الاخرى التي لا تتضمن في حساباتها الترابط الاككتروني. الهدف

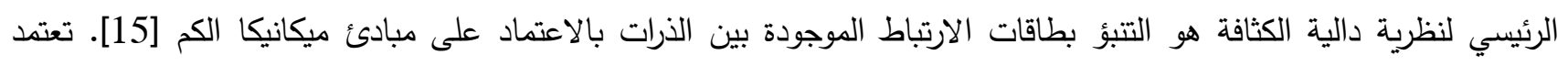

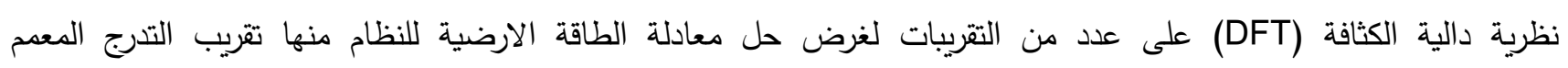
[16] الذي تم استخدامه في حساباتتا في هذا البحث. وتقريب الكثافة (Generalized Gradient Approximation) (GGA)

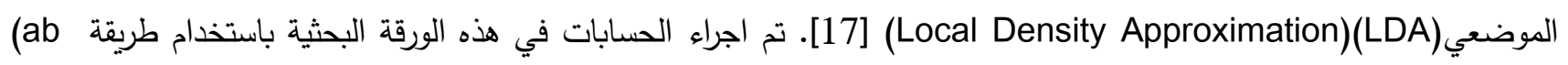
(Simulation بواسطة حزمة المحاكات initio) (Density Function Theory DFT) (الذي يتيح للباحث نمذجة (Density function Calculation on Molecular Dmol3) لبرنامج (modeling) بونة (Dackage) البنية الإلكترونية(electronic structure) وطاقة الجزيئات والمواد الصلبة والأسطح باستخدام نظرية دالية الكثافة (DFT) وينتج عن ذلك نتائج دقيقة للغاية مع الحفاظ على التكلفة الحسابية المنخفضة لطريقة (ab initio). طبقت هذه الشروط على وحدة الخلية 
الكبيرة للكرافين النقي (Pure Graphene) وخلية الكرافين المطعمة بذرة البورون(B) المفعلة بذرة النيكل (Ni) حيث أن مراحل الحسابات التي اجريت على تلك النماذج شملت, تهيئة البنية الفراغية ( Geometry Optimization) وبنية الحزم الاككترونية (Electronic Band Structure)

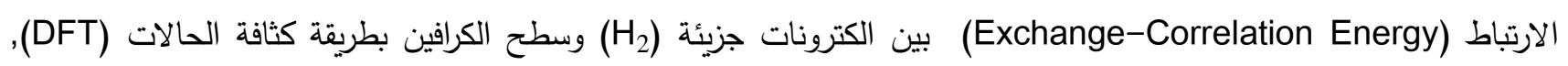

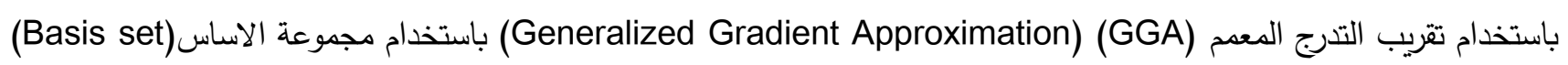
(Convergence وللحصول على دقة جيدة استخدمت قيم سماحية التقارب (Perdew-Burke-Ernzerhof) (PBE)

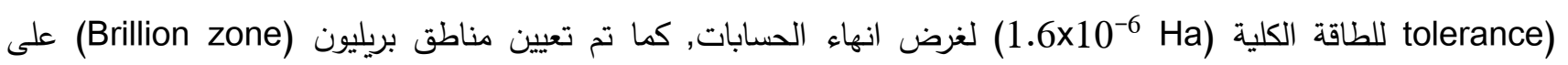
(2x2x1) للخلايا المدروسة, كما هو موضح في المخطط الانسيابي في الثكل (1). اجريت جميع العمليات الحسابية في البحث

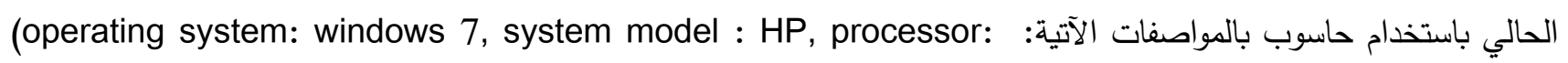
Intel(R) Cor i7, memory: $8 \mathrm{~GB}$, ).

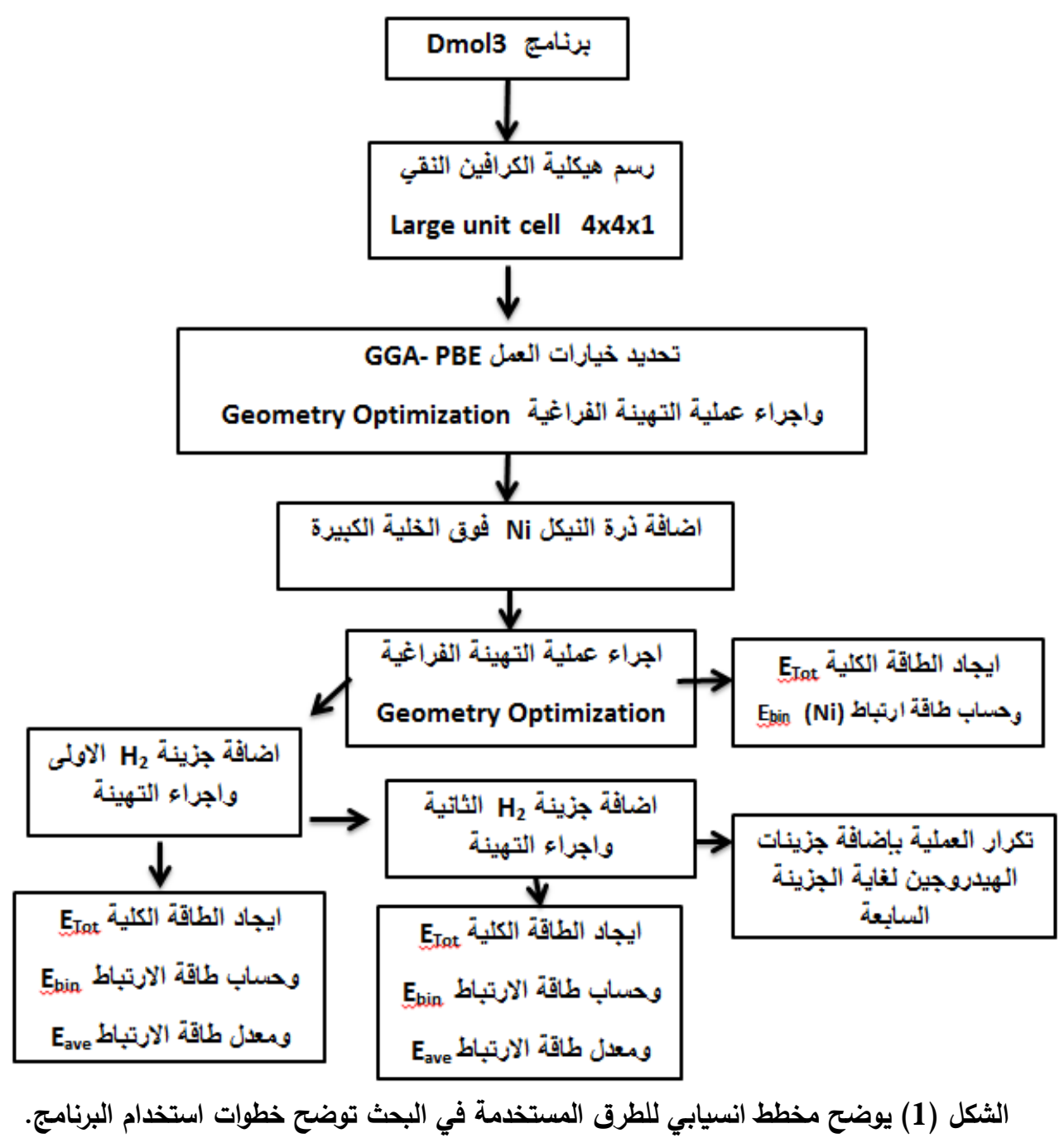

Results and Discussion النتائج و المناقشة

اولا. امتزاز ذرة النيكل(Ni)على سطح الكرافين النقي: 
تمت الحسابات على ذرة النيكل (Ni) بتطبيق الشروط الحدودية الدورية Periodic Boundary Condition) على وحدة

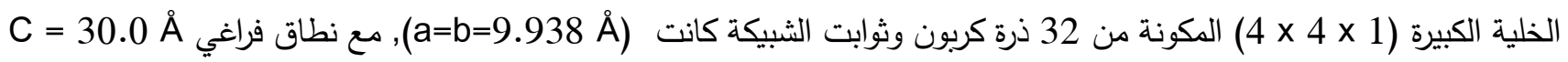
( ) لغرض تقليل تفاعل الطبقات المتداخلة. ولغرض معرفة الموقع المفضل لذرة النيكل (Ni) فوق سطح الكرافين اجريت عملية

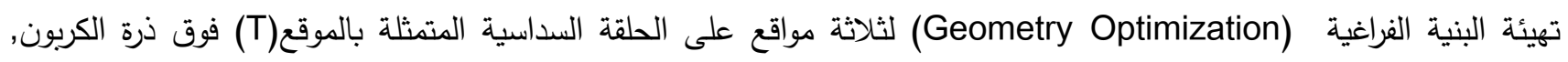

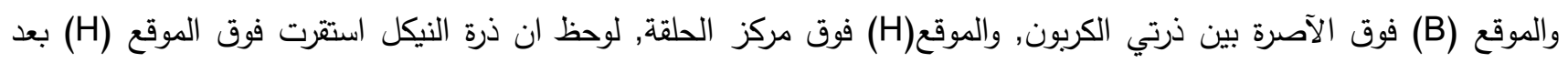
عملية التهيئة للمواقع الثلاث مما يعني ان الموقع (H) هو الموقع المفضل والاكثر استقرارا لذرة النيكل على سطح الكرافين كما

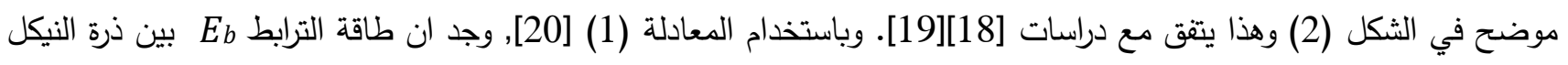

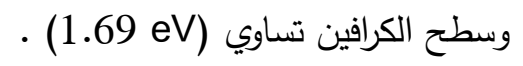

$$
E_{b}=E_{\text {graphene+metal }}-\left(E_{\text {graphene }}+n E_{\text {metal }}\right) / n
$$

حيث : Egraphene+metal: الطاقة الكلية للنظام مع n من ذرات النيكل المفعلة على سطحه, Egraphene : طاقة سطح الكرافين, Emetal

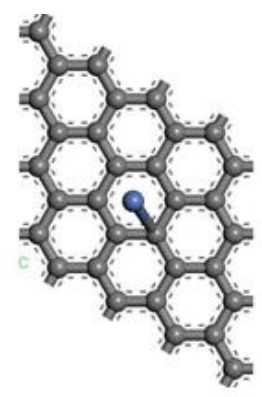

a) $\mathrm{H}$

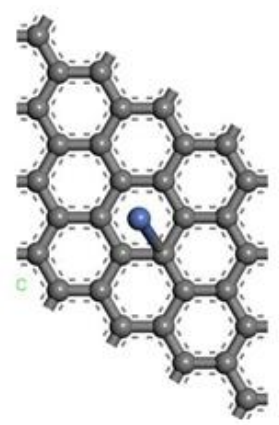

b) $\quad \mathrm{H}$

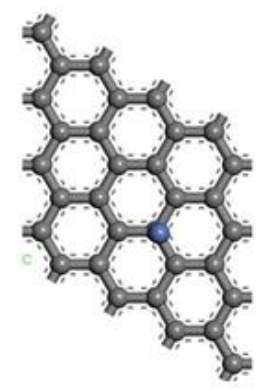

T

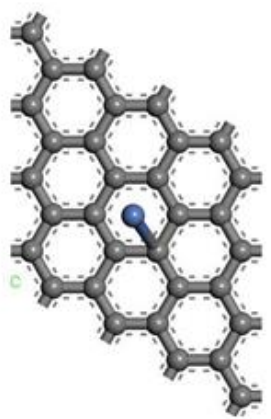

T

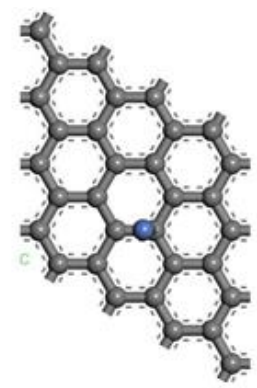

B

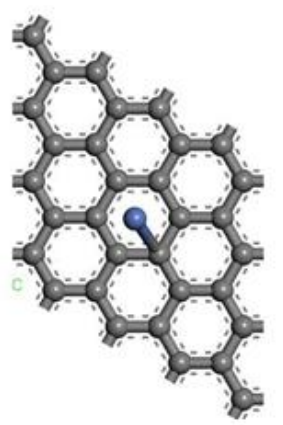

B

الثكل(2) يبين المواقع الثلاثة لذرة النيكل(باللون الازرق) على سطح الكرافين (a) قبل التهيئة (b) بعد التهيئة.

بناء على هذه الدراسة فان ذرات النيكل تميل الى التجمع فوق سطح الكرافين بسبب قوة التلاصق (a) (Cohesive Energy)

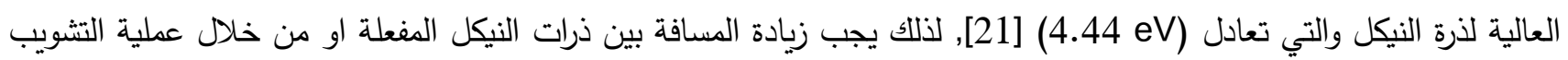

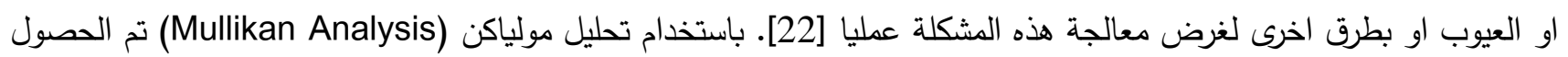

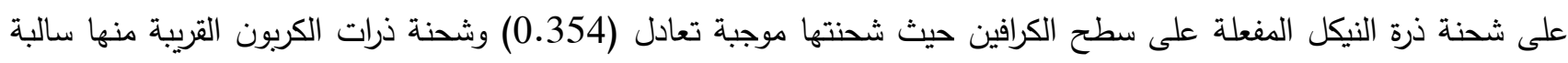
تتراوح بين (0.036) -032) كما هو في الثكل (3) مما يؤدي الى انتقال كبير للإلكترونات من الثحنة الموجبة لذرة النيكل الى دئ سطح الكرافين سالب الثحنة ليعزز الارتباط بين ذرة النيكل وسطح الكرافين [20]. 


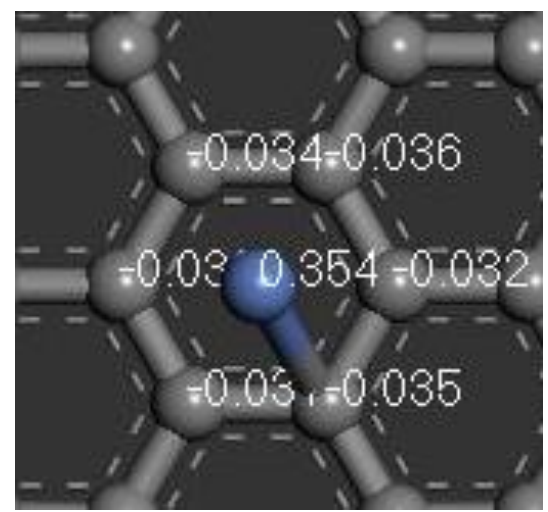

الثكل (3) يوضح توزيع الثحنات بين ذرة النيكل وذرات الكربون القريبة منها باستخدام تحليل موليكان.

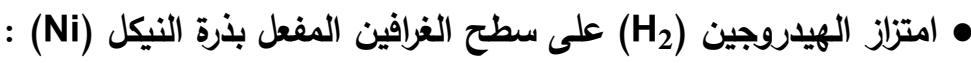
ان عملية امتزاز جزيئات الهيدروجين (Hi $)$ على سطح الكرافين المفعل بذرة النيكل (Ni) تمت بإضافة جزيئة هيدروجين واحدة وتهيئتها كل مرة و حساب طاقة ارتباطها (E)

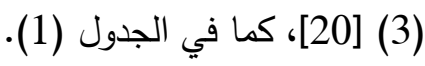

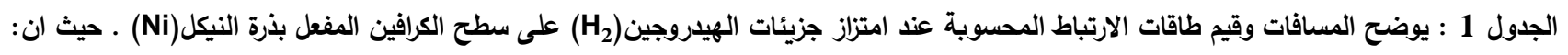
يمثل بعد جزيئة الهيروجين عن ذرة النيكل المفعلة على سطح الكرافين, (d $\left(\boldsymbol{d}_{\mathrm{H}_{2}-N i}\right)$ الكرافين, (

\begin{tabular}{|c|c||c||c||c||c||}
\hline $\begin{array}{c}\text { Number } \\
\text { of } \mathrm{H}_{2}\end{array}$ & $\begin{array}{c}d_{N i-c} \\
(\AA)\end{array}$ & $\begin{array}{c}d_{\mathrm{H}_{2}-\mathrm{Ni}} \\
(\AA)\end{array}$ & $\begin{array}{c}d_{\mathrm{H}_{2}-\mathrm{c}} \\
(\AA)\end{array}$ & $\begin{array}{c}\mathrm{E}_{\text {bind }(\mathrm{H})} \\
(\mathbf{e V})\end{array}$ & $\begin{array}{c}\mathrm{E}_{\text {average }} \\
\left(\mathbf{e V} / \mathbf{H}_{2}\right)\end{array}$ \\
\hline \hline 1 & 2.19 & 1.55 & 3.23 & 1.28 & 1.28 \\
\hline \hline 2 & 2.21 & 3.67 & 3.31 & 0.089 & 0.68 \\
\hline \hline 4 & 2.18 & 3.50 & 3.32 & 0.082 & 0.48 \\
\hline \hline 5 & 2.19 & 4.74 & 3.92 & 0.091 & 0.38 \\
\hline \hline 6 & 2.19 & 3.59 & 3.32 & 0.090 & 0.32 \\
\hline \hline 7 & 2.195 & 5.65 & 5.00 & 0.078 & 0.25 \\
\hline \hline
\end{tabular}

$E_{b_{2}}=-\left[E_{\text {graphene+metal }+m H_{2}}-E_{H_{2}}-E_{\text {graphene+metal }+(m-1) H_{2}}\right]$

$E_{\text {average }}=\frac{-\left[E_{\text {graphene }+ \text { metal }+m H_{2}}-\left(E_{\text {graphene }+ \text { metal }}+m E_{\mathrm{H}_{2}}\right)\right]}{m}$

حيث: الطاقة الكلية لسطح الكرافين المفعل بذرة الفلز (النيكل) مع عدد(m) من جزيء الهيدروجين, Egraphene+metal+(m-1) الهيدروجين, Egraphene+metal : طاقة سطح الكرافين الففعل بذرة الفلز (النيكل). 

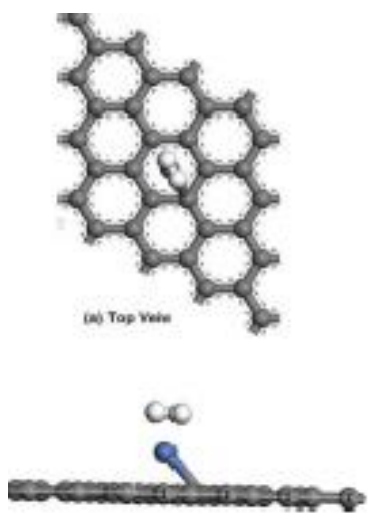

(a)side view

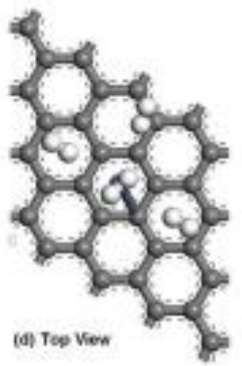

$180 \mathrm{co}$

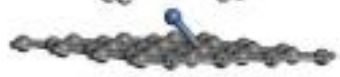

(d) side view

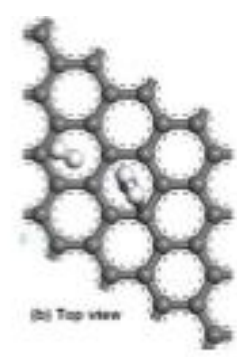

CO $\mathrm{CO}$

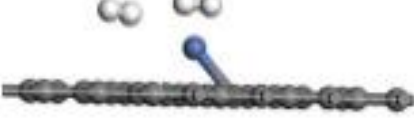

(b)side view

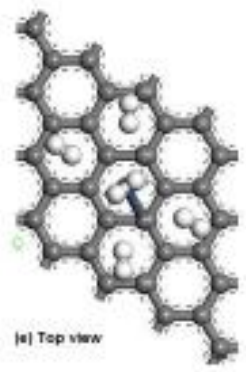

$10: a^{\circ}$

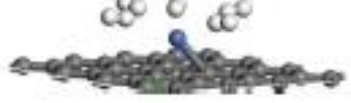

(e)side view
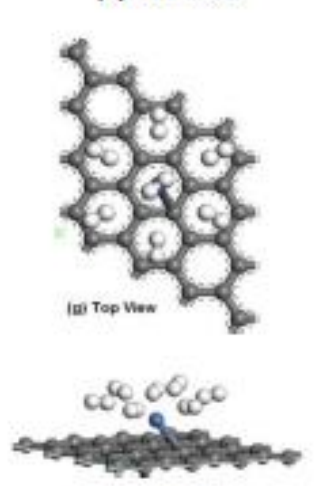

(g) side view
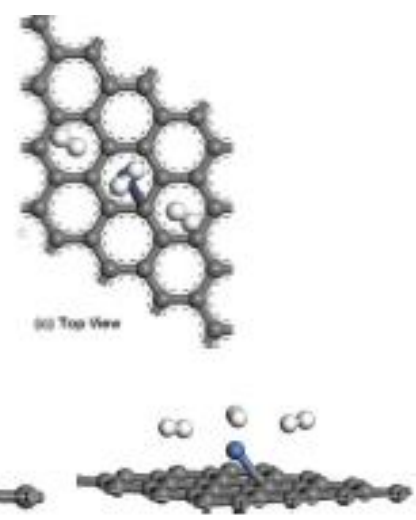

(c) side view
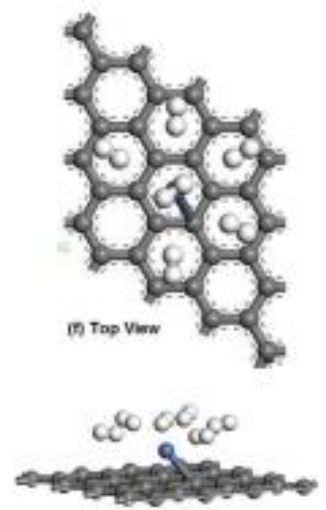

(f)side view

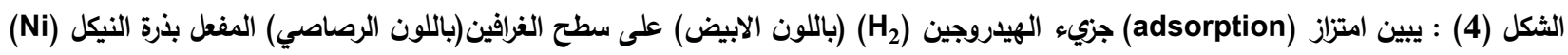

$$
\text { (باللون الازرق) (الانون الاند) }
$$

نلاحظ من الثكل (4) والجدول (1) ان اضافة جزيئة الهيدروجين الاولى على سطح الكرافين المفعل بذرة النيكل وقبل اجراء

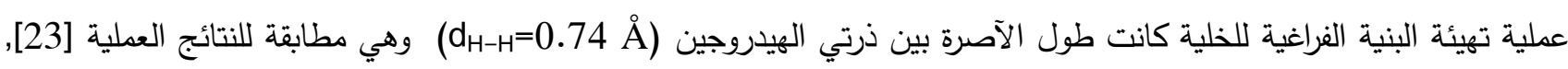

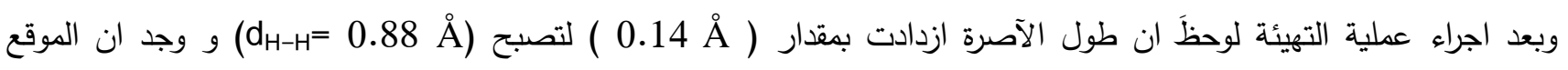
المفضل لجزيئة الهيدروجين الاولى هو اعلى الموقع (H) لسطح الكرافين وفوق ذرة النيكل المفعلة, اما جزيئات الهيدروجين المتبقية توزعت حول ذرة النيكل كما هو موضح في الشكل (4 a) فعند إضافة جزيء (H) الثاني واجراء التهيئة وجد انه استقر ايضاً على الثى

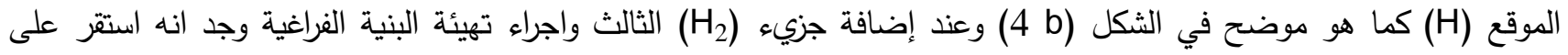
جانب الموقع (T) كما هو موضح في الثكل (C 4) وبإضافة جزيء (H (H) الرابع واجراء عملية التهيئة وجد انه استقر على الموقع فوق مركز الحلقة السداسية للغرافين كما موضح في الثكل (4 d d (H) وبإضافة جزيء (H) الخامس واجراء تهيئة البنية الفراغية 
وجد انه استقر على الموقع (H) كما هو موضح في الثكل (e 4) وعند إضافة جزيء (H2) السادس واجراء تهيئة البنية الفراغية وجد انه استقر بالقرب من الموقع (B) كما هو موضح في الثكل (4 f) وعند إضافة جزيء (H2) السابع واجراء تهيئة البنية

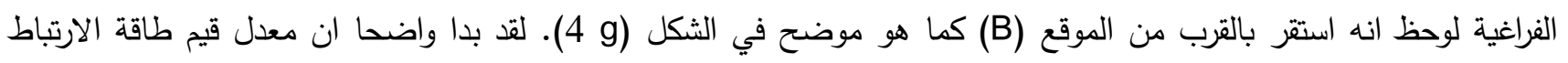
الموضحة بالجدول (1) والثكل (4) تتاسب عكسيا مع عدد جزيئات الهيدروجين الممتزة على سطح الكرافين, حيث تقل طاقة الارتباط عند زيادة عدد جزيئات الهيدروجين الممتزة على سطح الكرافين اذ تم تمثيلها بمخطط بياني بالشكل (5), هذه النتائج تتقق

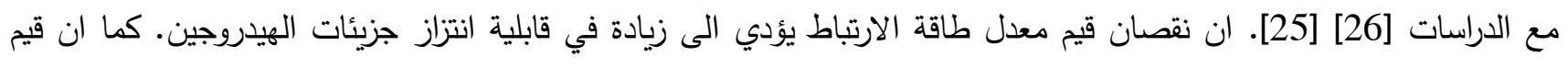

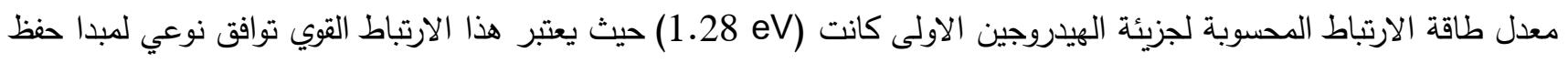

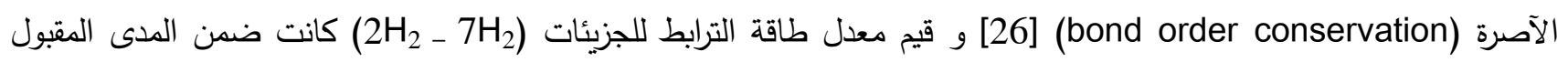
حيث تراوحت (24 eV (24) الاهرة

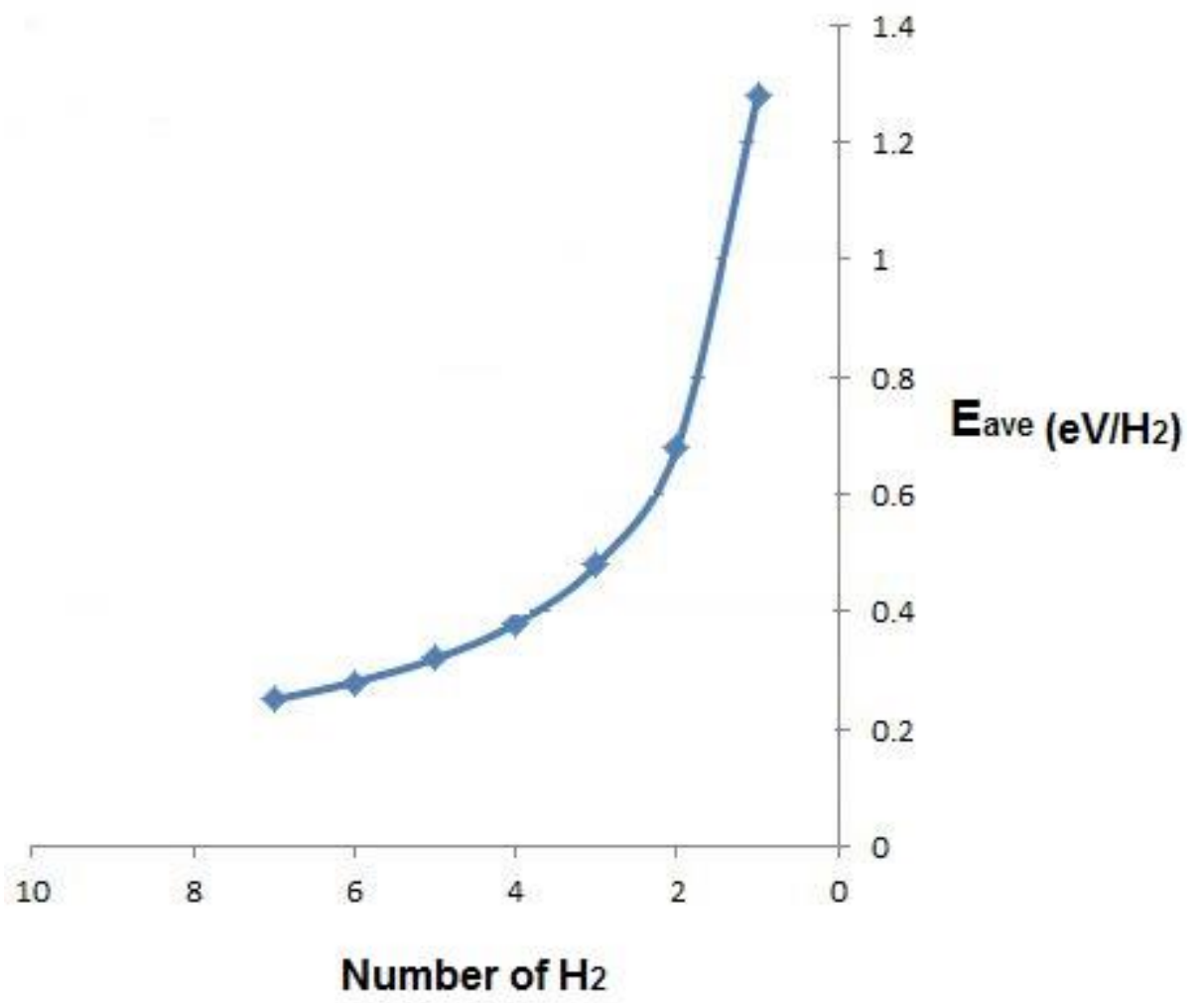

الثكل (5) : العلاقة العكسية بين قيم معدل طاقة الترابط (E average) وعدد جزيئات الهيل وجين (H) (H).

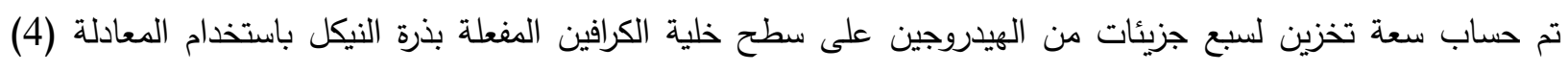

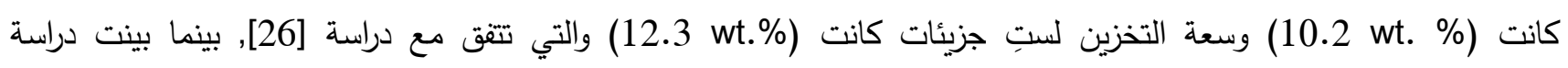
[Hong-Lae Park, Dong Su Yoo 2011)

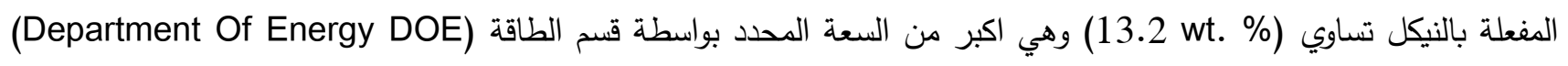

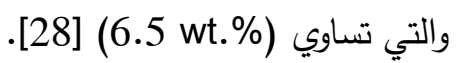

$$
H_{2}(w t \%)=\left[\frac{M_{H_{2}}}{M_{H_{2}}+M_{C}+M_{m}}\right] \times 100 \%
$$

حيث ان: وحدة خلية الكرافين, $M_{m}$ : كتلة الذرة المفعلة (ذرة النيكل). 
ثانيا. امتزاز ذرة النيكل (Ni) على سطح الكرافين المطعم بذرة البورون (B).

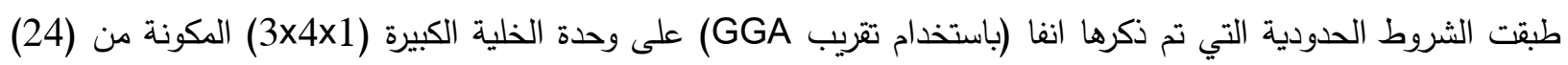

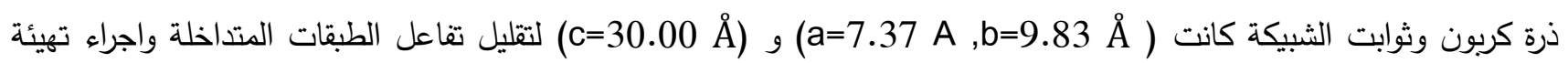

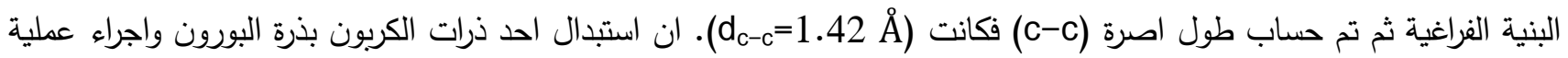

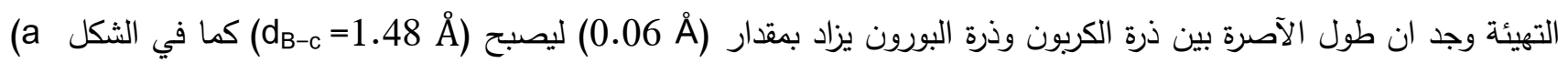

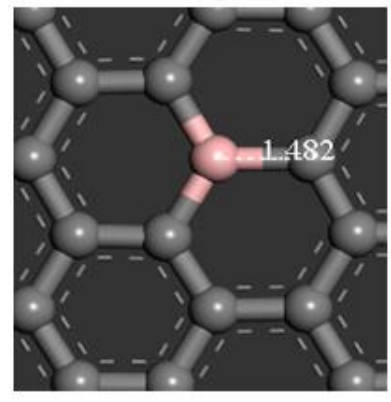

a)

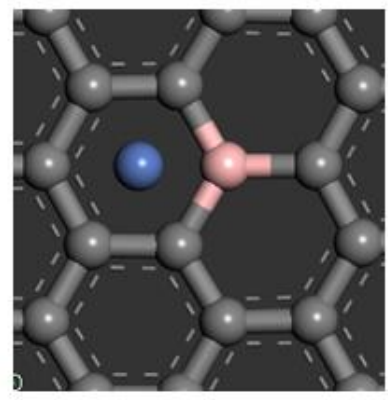

b)

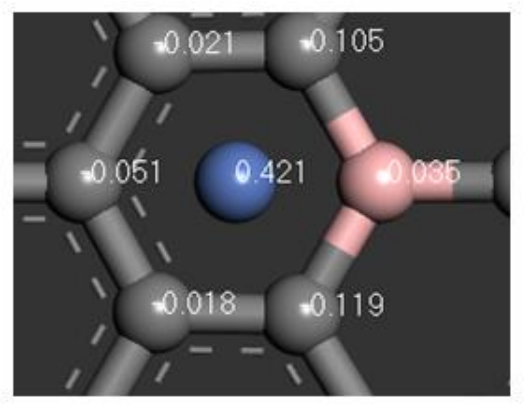

c)

الثكل (6) : (a) يبين طول

الآصرة لخلية الكرافين المطع بذرة البورون(B) (الوردي الفاتح). (b) امتزاز النيكل على سطح خلية الكرافين المطعم بذرة البورون.(c) توزيع الثحنات.

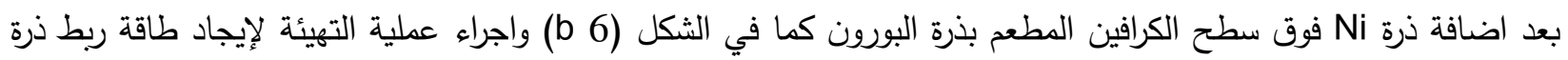
النيكل باستخدام العلاقة (1) وجد انها تساوي (2.76 eV), وهي اكبر من طاقة ارتباط ذرة النيكل على سطح الكرافين النقي ( 1.69 eV

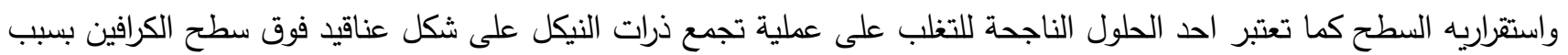

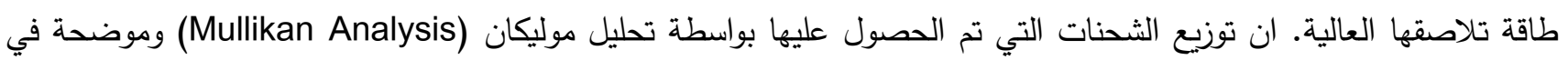
الشكل (6 c) تبين ان شحنة ذرة النيكل الموجبة تساوي (0.421) وشحنة ذرات الكرافين المطعمة بالبورون السالبة القريبة منها تتراوح بين (0.021-0.119), ينتج عن ذلك انتقال كبير للشحنات بين ذرة النيكل وسطح الكرافين يؤدي الى تعزيز الارتباط بينهما كما تم ذكره سابقا.

ثانيا: امتزاز جزيئات (H)(على سطح الغرافين المطعم بذرة (B) و المفعل بذرة(Ni).

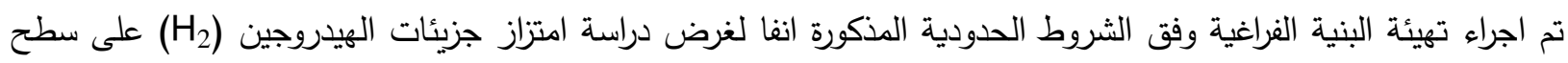
الكرافين المفعل بذرة النيكل والمطعم بذرة البورون وذلك بإضافة جزيئة الهيدروجين (H2) في كل مرة وتهيئتها وحساب طاقة ارتباطها (Everage) وفق العلاقة (2) ومعدل طاقة ارتباطها (2) وفق العلاقة (3),كما موضح في الجدول (2). 
الجدول 2 : يوضح المسافات وقيم طاقات الارتباط المحسوبة عند امتزاز جزيئات الهيدروجين(H) على سطح الكرافين المفعل بذرة النيكل(Ni) ) والمطعم بذرة البورون (B). حيث ان: الهيروجين عن ذرة النيكل المفعلة على سطح الكرافين, di-c

\begin{tabular}{||c||c||c||c||c||c|}
\hline \hline $\begin{array}{c}\text { Number of } \\
\mathrm{H}_{2}\end{array}$ & $\begin{array}{c}d_{N i-c} \\
(\AA)\end{array}$ & $\begin{array}{c}d_{\mathrm{H}_{2}-N i} \\
(\AA)\end{array}$ & $\begin{array}{c}d_{\mathrm{H}_{2}-c} \\
(\AA)\end{array}$ & $\begin{array}{c}\mathrm{E}_{\text {bind(H) }} \\
(\mathrm{eV})\end{array}$ & $\begin{array}{c}\mathrm{E}_{\text {average }} \\
\left(\mathrm{eV} / \mathrm{H}_{2}\right)\end{array}$ \\
\hline \hline 1 & 2.210 & 1.58 & 3.20 & 1.05 & 1.28 \\
\hline \hline 2 & 2.211 & 3.77 & 4.02 & 0.059 & 0.55 \\
\hline \hline 3 & 2.220 & 3.72 & 4.71 & 0.096 & 0.40 \\
\hline \hline 5 & 2.210 & 2.87 & 3.57 & 0.090 & 0.32 \\
\hline \hline 6 & 2.212 & 4.12 & 4.45 & 0.081 & 0.27 \\
\hline \hline 5 & 2.221 & 3.05 & 4.04 & 0.078 & 0.24 \\
\hline \hline 5
\end{tabular}




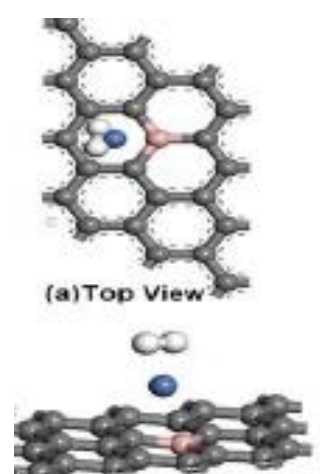

(a) Side View

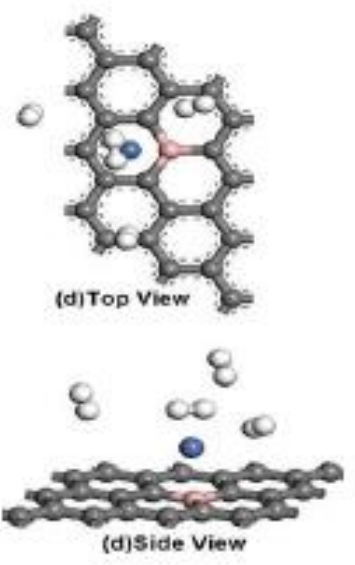

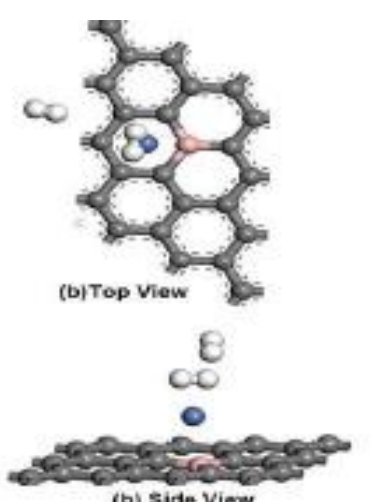

(b) side View
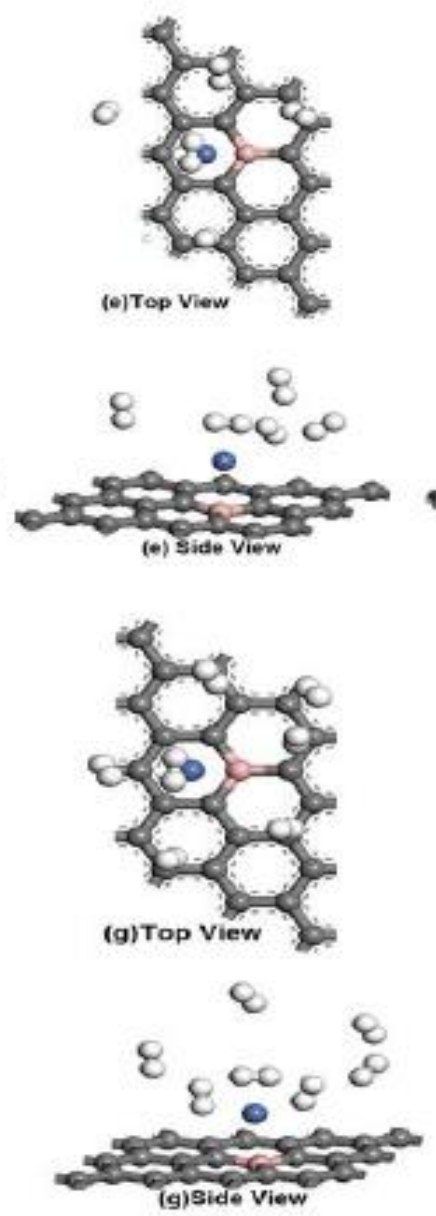
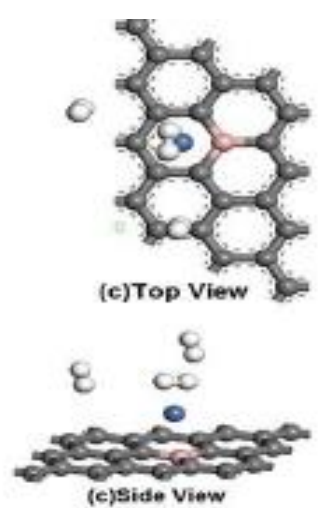

(c)Side View

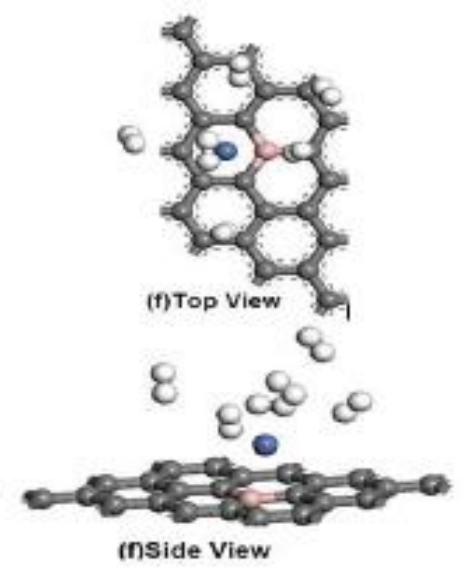

(nside View

الثكل (7) : يبين امتزاز (adsorption) جزيء الهيدرجين (Hion) على سطح الغرافين المفعل بذرة النيكل (Ni) والمطع بذرة البورون (B) .

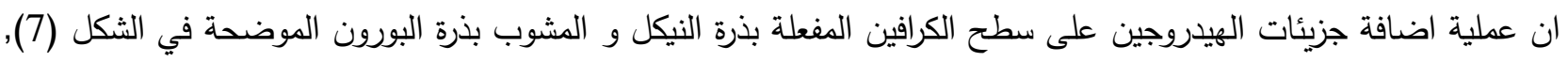
تمت من خلال اضافة جزيئة (H2) كل مرة واجراء تهيئة البنية الفراغية. عندما تم وضع جزيئة الهيدروجين الاولى كانت طول

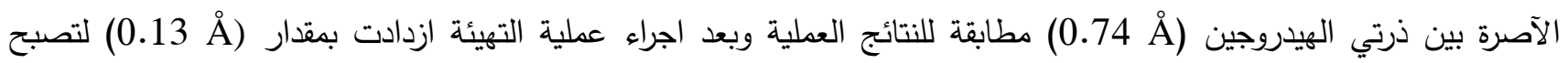

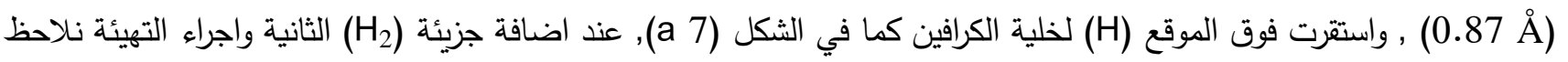

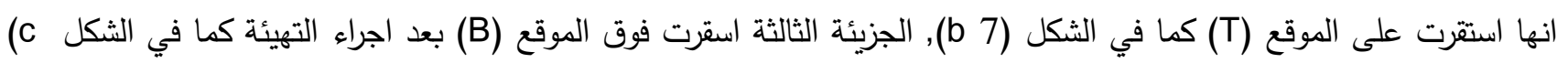
(7, عند اضافة الجزيئة الرابعة واجراء التهيئة نلاحظ انها استقرت بالقرب من الموقع (T) كما موضح في الشكل (d 7), الجزيئة

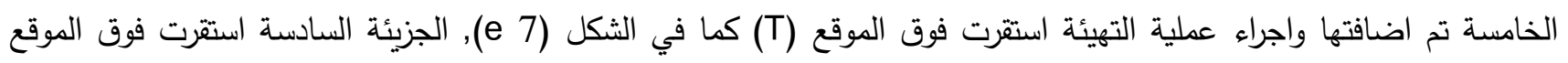
(B) بعد اجراء التهيئة كما في الثكل (7 7), اما الجزيئة السابعة استقرت فوق الموقع (T) بعد اجراء التهيئة كما في الثكل (7 g). 
من خلال الجدول (2) نلاحظ معدل طاقة الامتزاز لجزيئات الهيدروجين تقل بزيادة عدد الجزيئات الممتزة حيث ان هذا النقصان في طاقة الارتباط يزيد من قدرة انتزاز جزيئات الهيدروجين في الظروف المحيطة التي تم ذكرها آنفاً. الشكل (8) يوضح العلاقة العكسية بين معدل طاقة الامتزاز وعدد جزيئات الهيدروجين الممتزة.

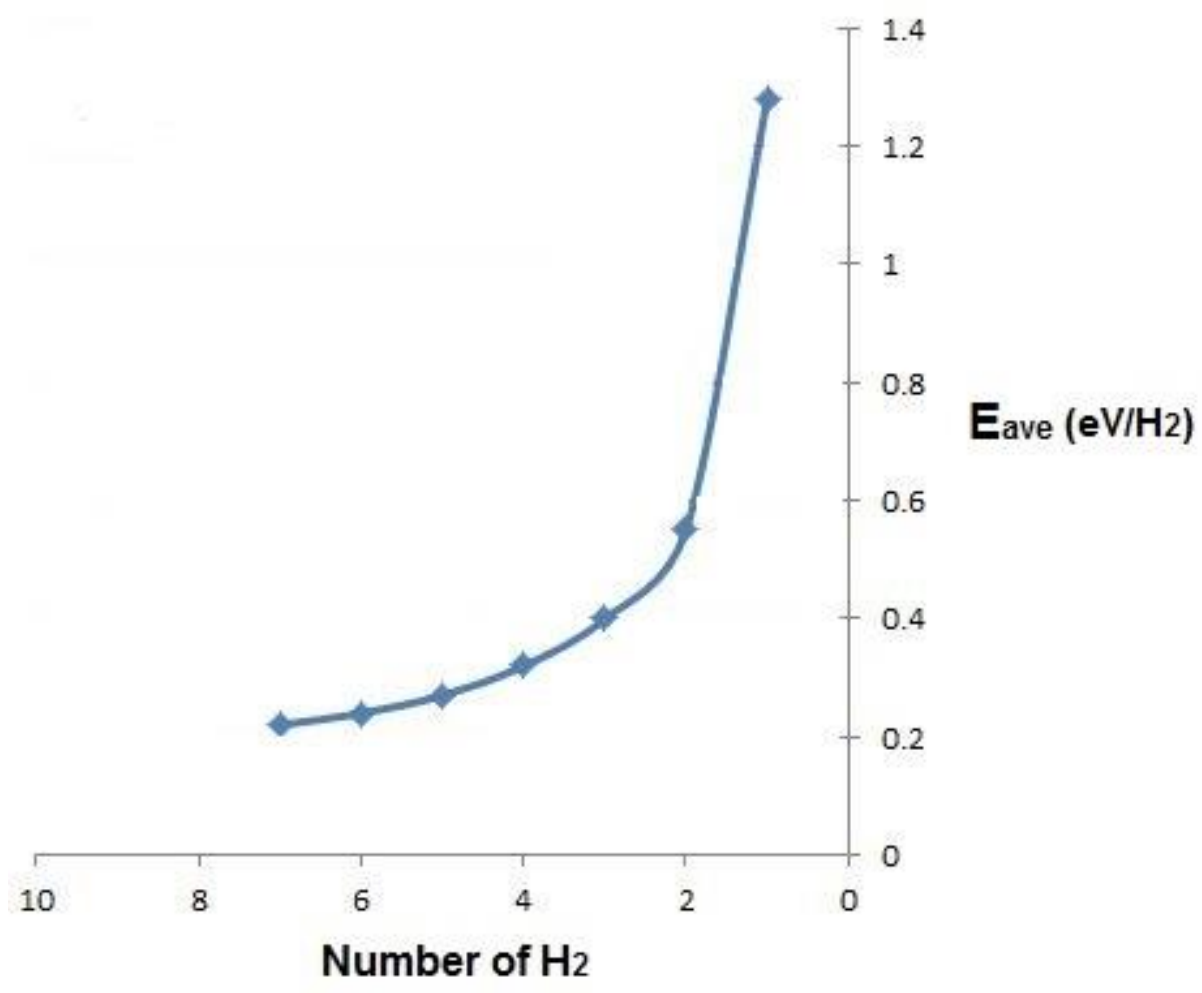

الثكل (8) : يوضح العلاقة العكسية بين عدد جزيئات الهيدروجين الممتزة و معدل طاقة الارتباط.

ان معدل طاقة الارتباط للجزيئة الاولى تساوي (1.28 eV) حيث يعد هذا الارتباط القوي توافق نوعي لمبدأ حفظ الآصرة كما تم

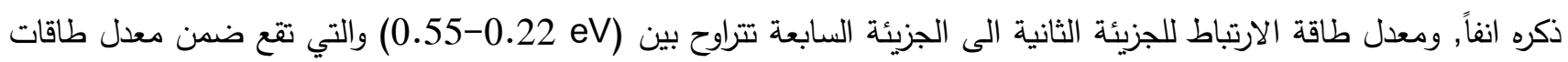

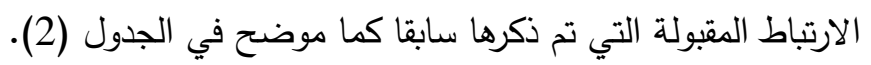
باستخدام العلاقة (4) تم حساب النسب الوزنية لخزن جزيئات الهيدروجين على سطح خلية الكرافين (3x4x1) المفعلة بذرة

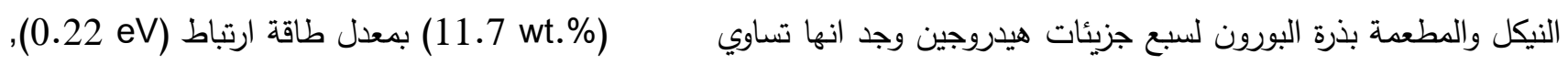

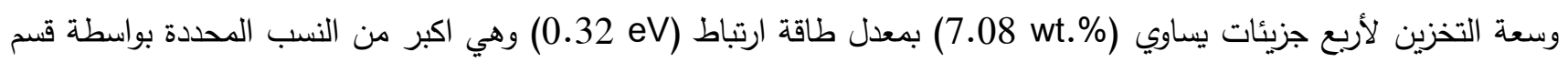
الطاقة (DOE) المشار اليها سابقا.

\section{Conclusions الاستنتاجات}

باستخدام حسابات (ab initio) بطريقة الكثافة الدالية (DFT) ومجموعة الاساس (PBE) بواسطة في برنامج (Dmol3), درسنا سلوك وطاقة ربط جزيئات الهيدروجين الممتزة على سطح خلية الكرافين النقي و المفعل بذرة النيكل مرة وسطح خلية الكرافين المطعمة بذرة البورون و المفعلة بذرة النيكل مرة اخرى. لوحظ ان طول الآصرة بين ذرتي الهيدروجين لكل جزيئة يزداد بحوالي

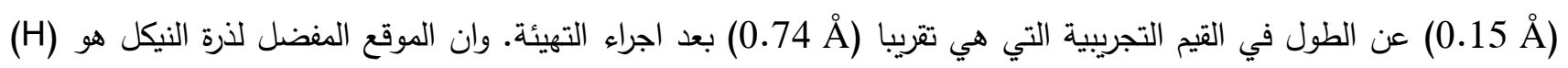
الموقع فوق مركز الحلقة السداسية لسطح الكرافين, ووجد أن طاقة الارتباط لذرة النيكل على سطح الكرافين النقي تساوي الا انها تساوي (2.76 eV) على سطح الكرافين المطعم بذرة البورون, وباستخدام تحليل موليكان وجد ان شحنة ذرة النيكل المفعلة على سطح الكرافين النقي تساوي (0.354) وشحنة ذرة النيكل المفعلة على سطح خلية الكرافين المطعمة بذرة البورون تساوي (0.421) اي ازدادت بمقدار (0.126). كما وجد ان جزيئات الهيدروجين تسلك السلوك ذاته عند امتزازها على سطح 


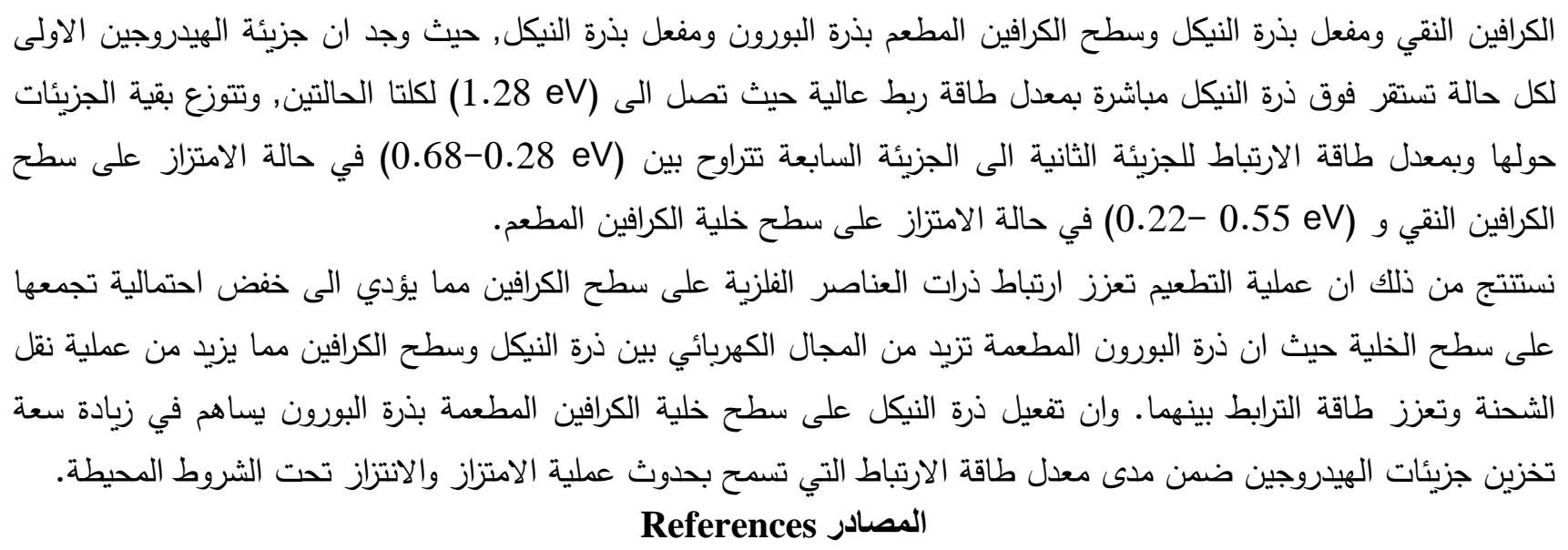

1. Sema Z. Baykara, "Hydrogen: A brief overview on its sources,production and environmental impact" international journal of hydrogen energy 1-10, (2018).

2. E. David."An overview of advanced materials for hydrogen storage" International scientific conference on achievements in mechanical and materials engineering .2005.

3. Meng $\mathrm{Ni}$, Michael K.H. Leung, DennisY.C. Leung, "Potential of renewable hydrogen production for energy supply in HongKong” International Journal of Hydrogen Energy ,31 $1401-1412$ (2006) .

4. A. PRAKASH ARANGA RAJU. "Production and Applications of Graphene and Its Composites" Thesis Faculty of Engineering and Physical Sciences The University of Manchester. 2015.

5. S. Shafraniuk. “Graphene Fundamentals, Devices, and Applications”. Taylor \& Francis Group, LLC. 2015.

6. S. Yadav, Z. Zhu, Chandra V. Singh. "Defect engineering of graphene for effective hydrogen storage". international journal of hydrogen energy 39(2014) 4981 e 4995 . P 4982.

7. Y. Chen, Jing Wang, Lihua Yuan, Meiling Zhang and Cairong Zhang. "Sc-Decorated Porous Graphene for High-Capacity Hydrogen Storage: First-Principles Calculations”. Materials 2017, 10, 894. P 1.

8. Y. Zhou, W. Chu, Fangli Jing, Jian Zheng, Wenjing Sun, Ying Xue. "Enhanced hydrogen storage on Li-doped defective graphene with B Substitution : A DFT study". Applied Surface Science 410 (2017) 166-176. P166.

9. Y. Wang, Yuhong Chen, Y. Wang. "Sodium decorated net-Y nanosheet for hydrogen storage and adsorption mechanism: A first-principles study". Chin. Phys. B Vol. 29, No. 1 (2020) 016801. P 1.

10. Z. M. Ao and F. M. Peeters. "Electric Field Activated Hydrogen Dissociative Adsorption to Nitrogen-Doped Graphene. J. Phys”. Chem. C 2010, 114, 14503-14509. P 14503.

11. E.G. Lewars, "Computational Chemistry", Springer International Publishing Switzerland 2016.

12. K. I. Ramachandran et al., “Computational Chemistry and Molecular Modeling”, Springer 2008.

13. Mudar A. Abdulsattar, "Size effects of semiempirical large unit cell method in comparison with nanoclusters properties of diamond-structured covalent semiconductors", Physica E 41, 1679 (2009).

14. N. Abuzaid. "Electronic Structure According to the Orbital Approximation and the Hartree-Fock Theory with Electron Correlation Methods jultika”.oulu.fi. 2016.

15. M.H.Cohen ,D .Frydel, K.BrukeandE .Engel,J. ”Chemical .Physics ”.Vol.113, p.2990. (2000)

16. Z. Wu and Ronald E. Cohen. "A More Accurate Generalized Gradient Approximation for Solids”, arXiv:cond-mat/0508004v3 [cond-mat.mtrl-sci] 24 Jan 2006. 
17. D. ,C. Young. “Computational Chemistry”. John Wiley \& Sons, Inc. 2001.

18. I. A, Yamamoto M, Asano H, Fujiwara K.” DFT calculation for adatom adsorption on graphene sheet as a prototype of carbon nanotube functionalization " Phs Conf ser 100:52087.

19. M. ,M, Andriotis AN, Froudakis CE. “curvature dependence of the metal catalyst atom interaction with carbon nanotubes walls” Chem Phys Lett 320:425-34. 2000.

20. Z. M. Ao and F. M. Peeters. "High-capacity hydrogen storage in Al-adsorbed graphene" . PHYSICAL REVIEW B 81, 2054062010.

21. C. Kittel. “Introduction to Solid State Physics, 8th edition”. Hoboken, NJ: John Wiley \& Sons, Inc, 2005.

22. V. Jain and Balasubramanian Kandasubramanian. "Functionalized graphene materials for hydrogen storage” Science+Business Media, LLC, part of Springer Nature 2019.

23. K. K Irikura "Experimental Vibrational Zero-Point Energies: Diatomic Molecules" J. Phys. Chem. Ref. Data 36(2), 389, 2007A.

24. Sigal, M.I. Rojas ,E.P.M. Leiva. "Interferents for hydrogen storage on a graphene sheet decorated with nickel: A DFT study". international journal of hydrogen energy 363537e3546. (2011).

25. S. Seenithurai, R. Kodi Pandyan, S. Vinodh Kumar, C. Saranya, M. Mahendran. “Lidecorated double vacancy graphene for hydrogen storage application: A first principles study". international journal of hydrogen energy 39,11016-11026, (2014).

26. S. Abdel Aal, A. S. Shalabi, K. A. Soliman. "High Capacity Hydrogen Storage in Ni Decorated Carbon Nanocone: A First-Principles Study" Journal of Quantum Information Science, 5, 134-149, 2015.

27. H. Lae Park, Dong Su Yoo, and Yong-Chae Chung. "Adsorption and Diffusion of Li and Ni on Graphene with Boron Substitution for Hydrogen Storage: Ab-initio Method”. Japanese Journal of Applied Physics 50 06GJ02, (2011).

28. J. Woo Lee, Hyun Seok Kim, Jai Young Lee, and Jeung Ku Kang. "Hydrogen storage and desorption properties of Ni-dispersed carbon nanotubes”. Applied Physics Litters 88, 143126 (2006). 